\title{
A two-zone model for the emission from RX J1713.7-3946
}

\author{
K. Moraitis and A. Mastichiadis
}

\author{
Department of Physics, University of Athens, Panepistimiopolis, 15783 Zografos, Athens, Greece \\ e-mail: kmorait@phys.uoa.gr
}

Received 30 May 2006 / Accepted 29 September 2006

ABSTRACT

\begin{abstract}
Aims. We study the acceleration and radiation of charged particles in the shock waves of supernova remnants using a recent version of the "box model". According to this, particles are accelerated in an energy-dependent region around the shock by the first order Fermi mechanism and lose energy through radiation.

Methods. The particle distribution function is obtained from a spatially averaged kinetic equation that treats the energy losses selfconsistently. There exists also a second population that consists of those particles that escape behind the shock where they also radiate. The energy distribution of this population is calculated in a similar manner.

Results. The application of the model to the supernova remnant RX J1713.7-3946, which was recently confirmed as a TeV source by HESS, shows that the X-ray emission can be attributed to electron synchrotron radiation while in $\gamma$-rays there are contributions from both electrons and protons, with protons playing the dominant role. Additionally, there are strong indications that particles diffuse in turbulence that has a Kolmogorov spectrum.
\end{abstract}

Key words. acceleration of particles - cosmic rays - radiation mechanisms: non-thermal - ISM: supernova remnants

\section{Introduction}

Supernova remnants (SNRs) have long thought to be the sites of galactic cosmic ray acceleration, at least up to energies close to that of the "knee" $\left(\sim 10^{15} \mathrm{eV}\right)$ of their spectrum (Krymskii 1977; Bell 1978; Blandford \& Ostriker 1978). Direct evidence for this came only during the last decade with the observation of non-thermal X-ray emission from SNRs. This can be best explained as synchrotron radiation by electrons that have been accelerated up to energies $\sim 100 \mathrm{TeV}$ (Reynolds \& Keohane 1999). Despite this success, evidence for the presence of a relativistic hadronic component is less certain. Accelerated hadrons in SNRs are expected to emit high energy gamma rays from the decay of neutral pions $\left(\pi^{0}\right)$ produced in proton-proton collisions (Dorfi 1991). These are best observed in the TeV regime (Drury et al. 1994), thus SNRs have been one of the main targets for the ground based Čerenkov detectors (for a review see Weekes et al. 2002). However, the above picture is complicated by the fact that the same high energy electrons that produce the synchrotron X-rays will Compton up-scatter the microwave photons to TeV energies (Mastichiadis 1996; Pohl 1996), contributing an extra component in this regime. Therefore one expects that any $\mathrm{TeV}$ detection of a SNR cannot be unequivocally attributed to the presence of a relativistic hadronic (or leptonic) component. Instead, careful modeling is required to determine the origin of these gamma-rays.

In modeling the multi-wavelength spectrum of SNRs, most authors assume for the particle energy distribution (electrons or protons) the form of a power-law with an exponential cut-off. The power-law is a natural consequence of Fermi acceleration while the exponential turnover is supposed to arise from energy losses or SNR age limitations. Despite the theoretical support for these assumptions, this approach is still phenomenological. On the other hand, the use of extensive numerical codes has proved a valuable tool for studying the acceleration, especially as they allow more complex phenomena, such as shock modification, to be taken into account (Ellison et al. 2001; Berezhko et al. 2002). The application of an analytical model however is interesting as it offers a good opportunity to obtain an insight into some of the basic principles of particle acceleration.

A development in the direction of an analytical approach to the problem of particle acceleration was made recently by Drury et al. (1999). This approach has expanded on the concept of the so-called "box" model for particle acceleration (for a review see Kirk et al. 1994) by assuming an energy-dependent size for the acceleration region. This inserts some modifications in the particle spectra and it is thus interesting to apply this model to fit the SNR multi-wavelength emission. A good starting point for this is the first SNR with confirmed TeV emission, RX J1713.7-3946 (Muraishi et al. 2000; Enomoto et al. 2002; Aharonian et al. 2004, 2006).

RX J1713.7-3946 (G347.3-0.5) is a shell-type supernova remnant that was discovered in X-rays during the ROSAT All Sky Survey (Pfeffermann \& Aschenbach 1996). It is a young SNR with an age of 2000 years, possibly associated with the supernova that took place in AD393 (Wang et al. 1997) and is located in the Galactic plane at a distance $\sim 1 \mathrm{kpc}$. The X-ray emission of RX J1713.7-3946 is purely non-thermal in nature (Koyama et al. 1997; Slane et al. 1999) and its X-ray morphology, as revealed by Chandra, is quite complicated, consisting of bright filaments and dark voids (Uchiyama et al. 2003) indicating a spatially inhomogeneous magnetic field and/or density of relativistic particles. In $\gamma$-rays, the remnant was originally observed by the CANGAROO telescope (Muraishi et al. 2000; Enomoto et al. 2002) and recently confirmed as a high energy source by HESS (Aharonian et al. 2004, 2006). The remnant also exhibits a weak radio emission (Lazendic et al. 2004). The broadband energy spectrum of RX J1713.7-3946 has been modeled by many authors, assuming either a leptonic (Uchiyama et al. 2003; Pannuti et al. 2003; Lazendic et al. 2004) and/or a 
hadronic origin (Enomoto et al. 2002; Aharonian et al. 2006; Berezhko \& Völk 2006) for the $\gamma$-ray emission, but without a conclusive result so far.

In this paper we apply the Drury et al. (1999) model to the supernova remnant RX J1713.7-3946 and draw some conclusions about its properties. In Sect. 2 we review the main characteristics of the model and obtain the distribution function of accelerated particles as well as that of the particles which have escaped behind the shock. In Sect. 3 we derive the expected multi-wavelength photon spectrum from these distributions and apply it to RX J1713.7-3946. In Sect. 4 we summarize and discuss our results.

\section{The acceleration model}

Charged particles interacting with the shock wave of a supernova remnant can be accelerated to high energies through first order Fermi acceleration. Alfvén waves in the background medium scatter the charged particles, changing their direction but not their energy. Particles are thus confined to a region around the shock and are forced to cross it many times. The difference in the gas velocity upstream and downstream results in the statistical acceleration of particles after many shock crossings. While confined around the shock, particles lose energy since they emit radiation. At the same time a fraction of them escapes behind the shock towards the interior of the remnant where it also radiates. We next calculate the distribution function of these two populations when the accelerated particles are electrons as well as when they are protons. We denote quantities in the acceleration region with the subscript 1 and in the escape region with the subscript 2.

Although the shock in a SNR is spherical we can treat the acceleration in the plane shock geometry as long as the size of the acceleration region is much smaller than the SNR radius. We assume the presence of a uniform magnetic field that is normal to the shock. We also adopt the test particle approximation, i.e. we assume zero particle pressure. With these assumptions, acceleration takes place in a region around the shock of size $L=L_{\mathrm{u}}+L_{\mathrm{d}}=K_{\mathrm{u}} / U_{\mathrm{u}}+K_{\mathrm{d}} / U_{\mathrm{d}}$ where $K_{\mathrm{u}(\mathrm{d})}, U_{\mathrm{u}(\mathrm{d})}$ are the upstream (downstream) diffusion coefficient and gas velocity respectively. Assuming that the diffusion coefficient is continuous across the shock, i.e. $K_{\mathrm{u}}=K_{\mathrm{d}}=K$, the size of the acceleration region can be further written $L=K(r+1) / U_{\mathrm{u}}$ where $r=U_{\mathrm{u}} / U_{\mathrm{d}}$ is the compression ratio of the shock. Following Drury et al. (1999) we let the diffusion coefficient be momentum-dependent and adopt a general power-law dependence $K(p)=\kappa p^{\delta}$ with $\delta>0$. This choice makes the mathematical analysis easier but also covers some physically interesting cases. For example, the case $\delta=1$ corresponds to Bohm diffusion, $\delta=1 / 3$ to diffusion from Alfvén waves with a Kolmogorov spectrum of turbulence and $\delta=1 / 2$ to a Kraichnan spectrum. The same power-law dependence holds for the size of the acceleration region $L(p)=\kappa(r+1) p^{\delta} / U_{\mathrm{u}}$ as well as the acceleration timescale which is given by the relation

$t_{\text {acc }}(p)=\frac{3 L(p)}{U_{\mathrm{u}}-U_{\mathrm{d}}}$

If the accelerated particles are electrons they also experience energy losses through synchrotron radiation and/or inverse Compton scattering (IC). In the case where the latter is in the Thomson limit, we can write for the particle total losses $\dot{p}=$ $-\alpha_{1} p^{2}$, where

$\alpha_{1}=\frac{4}{3} \frac{\sigma_{\mathrm{T}}\left(U_{\mathrm{B}_{1}}+U_{\mathrm{ph}}\right)}{m^{2} c^{2}}$ is the sum of the energy densities of the magnetic field $U_{\mathrm{B}_{1}}$ and the target photon field $U_{\mathrm{ph}}, \sigma_{\mathrm{T}}$ is the Thomson cross section, $m$ the electron mass and $c$ the speed of light. If there were no energy losses, particles would escape from the acceleration region with the downstream flow at the bulk velocity $U_{\mathrm{d}}$. The presence of losses however generates a downward flux in momentum space and when combined with the increasing size of the acceleration region gives an additional escape process. The velocity of escape thus increases and the escape timescale is given by

$t_{\mathrm{esc}}(p)=\frac{L(p)}{U_{\mathrm{d}}+\alpha_{1} p^{2} \frac{\mathrm{d} L_{\mathrm{d}}}{\mathrm{d} p}}$

The second term in the denominator is the addition of the Drury et al. (1999) model to the standard box model and vanishes for a constant diffusion coefficient or no energy losses.

The evolution of the spatially averaged isotropic distribution function of particles at the shock front, $N_{1}(p, t)$, is governed by the continuity equation

$\frac{\partial N_{1}}{\partial t}+\frac{\partial}{\partial p}\left[N_{1}\left(\frac{p}{t_{\mathrm{acc}}(p)}-\alpha_{1} p^{2}\right)\right]+\frac{N_{1}}{t_{\mathrm{esc}}(p)}=Q(p, t)$.

The first term in brackets describes acceleration at the momentum dependent rate $t_{\text {acc }}^{-1}$ and the second the synchrotron-type energy losses. The last term in the left hand side describes particle escape at the momentum dependent rate $t_{\mathrm{esc}}^{-1}$ and the source term $Q(p, t)=Q_{0} \delta\left(p-p_{0}\right) \vartheta(t)$ represents injection of particles at low momenta $p_{\mathrm{o}}$ with the constant rate $Q_{\mathrm{o}} ; \delta(x)$ and $\vartheta(x)$ are the Dirac delta and Heavyside (or unit-step) functions respectively.

From the equation describing the evolution of a particle's momentum

$\frac{\mathrm{d} p}{\mathrm{~d} t}=\frac{p}{t_{\mathrm{acc}}(p)}-\alpha_{1} p^{2}$

it follows that particles can be accelerated up to the momentum where the acceleration and cooling timescales are equal, namely

$p_{\max }=\left[\frac{(r-1) U_{\mathrm{u}}^{2}}{3 r(r+1) \alpha_{1} \kappa}\right]^{\frac{1}{\delta+1}}$.

Particles reach this maximum allowed momentum if given enough time, otherwise their momentum is determined from the solution of Eq. (5). This equation can be solved analytically for the time a particle of initial momentum $p_{\mathrm{o}}$ needs to reach momentum $p$ and the solution is

$t_{1}(p)=t_{\mathrm{cool}, 1}\left[\frac{x^{\delta}}{\delta}{ }_{2} F_{1}\left(\frac{\delta}{\delta+1}, 1 ; 1+\frac{\delta}{\delta+1} ; x^{\delta+1}\right)\right]_{p_{0} / p_{\max }}^{p / p_{\max }}$

where ${ }_{2} F_{1}$ is the hypergeometric function and $t_{\mathrm{cool}, 1}=$ $\left(\alpha_{1} p_{\max }\right)^{-1}$ is the minimum cooling timescale. The inverse function of $t_{1}(p)$ gives the maximum momentum of a particle at time $t$ and is denoted as $p_{1}(t)$. This maximum momentum increases with time until energy losses become important and the acceleration saturates at $p_{\max }$ as can be seen in Fig. 1. The time required for this (in units of $t_{\mathrm{cool}, 1}$ ) is a decreasing function of the index $\delta$.

The solution of Eq. (4) for the distribution function is then

$$
\begin{aligned}
N_{1}(p, t)= & \frac{Q_{\mathrm{o}} t_{\mathrm{a}, \mathrm{o}}}{p_{\mathrm{o}}}\left(\frac{p}{p_{\mathrm{o}}}\right)^{-s_{1}} \\
& \times\left[1-\left(\frac{p}{p_{\max }}\right)^{\delta+1}\right]^{-b}\left[1-\left(\frac{p_{\mathrm{o}}}{p_{\max }}\right)^{\delta+1}\right]^{b-1}
\end{aligned}
$$




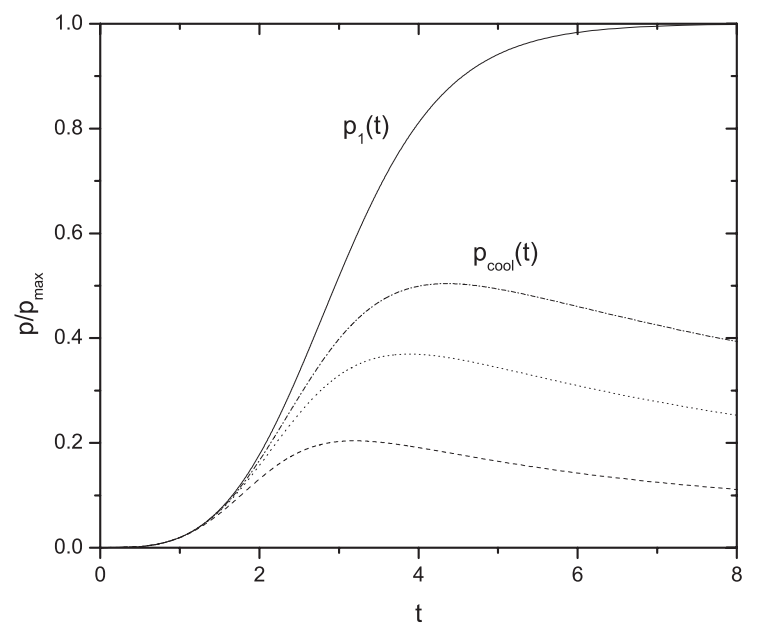

Fig. 1. The evolution of the upper limit of the particle distribution function $p_{1}(t)$ (solid line) for parameters $p_{0}=0, \delta=0.3$ and $B_{1}=$ $10 \mu \mathrm{G}$. Time is measured in units of the cooling timescale $t_{\text {cool, } 1}=$ $2200\left(p_{\max } c / 50 \mathrm{TeV}\right)^{-1} \mathrm{yr}$. The evolution of the cooling momentum $p_{\text {cool }}(t)$ for the cases $B_{2}=10 \mu \mathrm{G}$ (dashed line), $B_{2}=5 \mu \mathrm{G}$ (dotted line) and $B_{2}=2 \mu \mathrm{G}$ (dash-dotted line) is also shown.

in the momentum range $p_{\mathrm{o}}<p<p_{1}(t)$ and zero otherwise; $t_{\mathrm{a}, \mathrm{o}}$ is the minimum acceleration timescale, $t_{\mathrm{acc}}\left(p_{\mathrm{o}}\right)$, and the index $b=\frac{1}{\delta+1}\left(\frac{r-4}{r-1}+\frac{\delta}{r+1}\right)$. The accelerated particles form a power-law of index $s_{1}=\frac{r+2}{r-1}-\delta$, smaller by $\delta$ than the index predicted by the standard box model that extends from the injection momentum $p_{\mathrm{o}}$ up to the maximum momentum $p_{1}(t)$. Given enough time the particle distribution function reaches steady-state and is then given by Eq. (8) in the momentum range $p_{\mathrm{o}}<p<p_{\max }$. At steady-state the power-law is modified since the first term in brackets in Eq. (8) is zero at $p=p_{\max }$ and a pile-up or a cut-off of particles forms depending on whether the index $b$ is positive or negative.

Particles that escape towards the interior of the remnant play the most important role in the emission (Ball \& Kirk 1992; Aharonian \& Atoyan 1999) and we calculate their energy distribution. The distribution function of escaping particles obeys a similar equation to Eq. (4) where now the source term is the escape rate of accelerated particles while there are no acceleration or escape terms

$\frac{\partial N_{2}}{\partial t}-\frac{\partial}{\partial p}\left(\alpha_{2} p^{2} N_{2}\right)=\frac{N_{1}(p, t)}{t_{\mathrm{esc}}(p)}$.

The energy losses have the same form as in the acceleration zone (see Eq. (2)) but depend on the magnetic field of the escape zone, $B_{2}$. The solution of Eq. (9) can be easily obtained numerically (and semi-analytically) but we prefer a simplified analytic approach. If the energy losses are negligible the solution is simply the escape rate integrated over time

$$
\begin{aligned}
N_{2}(p, t) & =\frac{Q_{\mathrm{o}}\left(t-t_{1}(p)\right)}{p_{\mathrm{o}}}\left(\frac{p}{p_{\mathrm{o}}}\right)^{-s_{2}}\left[1-\left(\frac{p}{p_{\max }}\right)^{\delta+1}\right]^{-b} \\
& \times\left[1-\left(\frac{p_{\mathrm{o}}}{p_{\max }}\right)^{\delta+1}\right]^{b-1}\left[\frac{3}{r-1}+\delta \frac{r}{r+1}\left(\frac{p}{p_{\max }}\right)^{\delta+1}\right],
\end{aligned}
$$

which, for $p \ll p_{1}(t)$, is a power-law with the standard box model index $s_{2}=s_{1}+\delta=\frac{r+2}{r-1}$. This result remains valid when losses are present but only for those particles that have not had enough time to cool, i.e. those with momentum less than

$p_{\text {cool }}(t)=\frac{p_{1}(t)}{1+\alpha_{2} p_{1}(t) t}$.

This equation describes the evolution of the momentum of the highest energy particles as they cool in the escape zone and is shown in Fig. 1. On the other hand, particles with momentum exceeding $p_{\text {cool }}(t)$ had the necessary time to cool and their distribution is steeper than the injected one by one power of $p$, i.e.

$N_{2, \text { cool }}(p, t) \simeq N_{2}(p, t) \frac{p_{\text {cool }}(t)}{p}$.

The escaping particles thus have a broken power-law distribution, given by Eq. (10) for $p<p_{\text {cool }}(t)$ and by Eq. (12) for $p_{\text {cool }}(t)<p<p_{1}(t)$. The break occurs at the cooling momentum that depends on the magnetic field of the escape region. The extent of the cooled part of the particle distribution, $p_{1}(t)-p_{\text {cool }}(t)$, is greater for higher values of $B_{2}$ as is shown in Fig. 1. The steady-state escape distribution consists only of cooled particles and hence has a power-law index equal to $s_{2}+1$.

We next consider the case when the accelerated particles are protons. Since protons lose substantial energy only through the production of pions and the corresponding cooling timescale is much larger than the age of the remnant, $t_{\mathrm{pp}} \simeq 5 \times 10^{15} \mathrm{~s} \gg$ $t_{\mathrm{SN}} \simeq 2000 \mathrm{yr}$, we can neglect their energy losses. We thus solve Eqs. (4) and (9) without the energy loss term and for a source function that provides protons of momentum $\bar{p}_{\mathrm{o}}$ at the rate $\bar{Q}_{\mathrm{o}}$. The proton energy distribution in the acceleration zone has then the simple form

$N_{1}(p, t)=\frac{\bar{Q}_{\mathrm{o}} \bar{t}_{\mathrm{a}, \mathrm{o}}}{\bar{p}_{\mathrm{o}}}\left(\frac{p}{\bar{p}_{\mathrm{o}}}\right)^{-s_{1}}$,

in the momentum range $\bar{p}_{\mathrm{o}}<p<\bar{p}_{1}(t)$ and $\bar{t}_{\mathrm{a}, \mathrm{o}} \equiv t_{\mathrm{acc}}\left(\bar{p}_{\mathrm{o}}\right)$. The upper limit of the proton distribution follows from the solution of Eq. (5) with $\alpha_{1}=0$ and is given by

$\bar{p}_{1}(t)=\bar{p}_{\mathrm{o}}\left(1+\delta \frac{t}{\bar{t}_{\mathrm{a}, \mathrm{o}}}\right)^{\frac{1}{\delta}}$.

The distribution in the escape region is

$N_{2}(p, t)=\frac{3}{r-1} \frac{\bar{Q}_{\mathrm{o}}\left(t-\bar{t}_{1}(p)\right)}{\bar{p}_{\mathrm{o}}}\left(\frac{p}{\bar{p}_{\mathrm{o}}}\right)^{-s_{2}}$,

in the momentum range $\bar{p}_{\mathrm{o}}<p<\bar{p}_{1}(t)$, where again $\bar{t}_{1}(p)$ is the inverse function of $\bar{p}_{1}(t)$. We note that the power-law indices $s_{1}$, $s_{2}$ are the same as in the leptonic case.

\section{Application to the supernova remnant RX J1713.7-3946}

We now apply the model developed in the previous section to the supernova remnant RX J1713.7-3946. We first consider the case where only electrons are responsible for the observed emission; we neglect the possible contribution from a hadronic component. As discussed in Sect. 2 there exist two relativistic electron populations, one in the acceleration region and one in the escape region behind the shock. These electrons will produce synchrotron radiation in the magnetic field of the two regions, assumed equal for simplicity. Electrons will also Compton up-scatter the photons of the cosmic microwave and infrared background radiation fields which are the same in both zones. For the microwave 


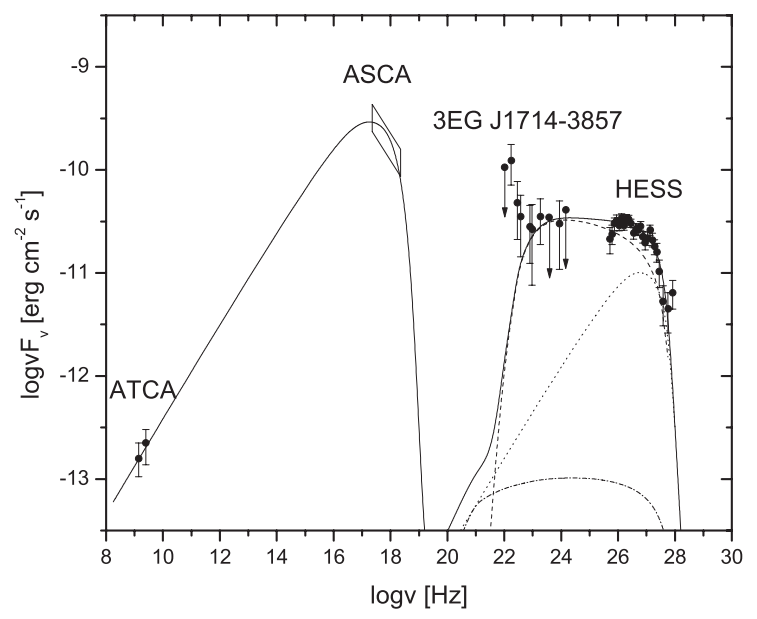

Fig. 2. The multi-wavelength spectrum of RX J1713.7-3946 together with the best-fit model with parameters given in the text. We also show the GeV upper limits from the nearby source 3EG J1714-3857. The dashed line represents the emission from neutral pions, the dotted line from IC scattering and the solid line is the total emission. Also plotted is the bremsstrahlung emission for the average ambient density $n=$ $1 \mathrm{~cm}^{-3}$ as the dash-dotted line.

photon field we take the standard values of temperature $T_{\mathrm{MW}}=$ $2.7 \mathrm{~K}$ and energy density $U_{\mathrm{MW}}=0.25 \mathrm{eV} \mathrm{cm}^{-3}$ while for the infrared field we assume $T_{\mathrm{IR}}=25 \mathrm{~K}$ and $U_{\mathrm{IR}}=0.05 \mathrm{eV} \mathrm{cm}^{-3}$ (Aharonian et al. 2006). In the possible emission processes we also include non-thermal bremsstrahlung radiation and assume for the ambient hydrogen density the average value $n \sim 1 \mathrm{~cm}^{-3}$.

The photon spectrum is derived by folding the two electron distribution functions with the single particle emissivity for the synchrotron, IC scattering and bremsstrahlung processes and thus depends on the same parameters that determine the electron distribution function in the acceleration and escape zones. These parameters are the compression ratio of the shock $r$, the index of the diffusion coefficient $\delta$, the magnetic field $B$, the maximum possible electron energy $E_{\mathrm{e}, \max }=p_{\max } c$ and the normalization constant $Q_{\mathrm{o}}$ and can be determined from the fit of the model to the observed spectrum. The injection momentum of electrons does not alter the spectrum significantly and so we take the arbitrary value $p_{\mathrm{o}}=5 \mathrm{MeV} / \mathrm{c}$. The photon spectrum is calculated at the present day, $t=t_{\mathrm{SN}}$.

The multi-wavelength spectrum of RX J1713.7-3946 is shown in Fig. 2. Radio data from the northwest part of the remnant taken with ATCA (Lazendic et al. 2004) are shown here multiplied by a factor of 2 to cover the whole remnant. Likewise, the X-ray data of ASCA (Enomoto et al. 2002) are multiplied by a factor of 4 so that the total X-ray flux from the remnant is obtained (Tomida 1999). The $\mathrm{TeV}$ data are the recent observations of HESS for the entire remnant (Aharonian et al. 2006). We also plot the EGRET data of the nearby source 3EG J1714-3857, since as emphasized by Reimer \& Pohl (2002) they set an upper limit to the GeV emission of RX J1713.7-3946.

We first examine a purely leptonic model in which the X-ray emission is due to synchrotron radiation and the $\gamma$-ray emission is due to IC scattering. From the observational data plotted in Fig. 2 we deduce that the X-ray emission peaks at $\varepsilon_{\max }^{\mathrm{X}} \sim 1 \mathrm{keV}$ and the $\gamma$-ray emission at $\varepsilon_{\max }^{\mathrm{TeV}} \sim 1 \mathrm{TeV}$. Since the peak frequencies scale as $E_{\mathrm{e}, \max }^{2} B$ and $E_{\mathrm{e}, \max }^{2}$ respectively (IC scattering takes place mostly in the Thomson regime), we find that the magnetic field should be $B \sim 40 \mu \mathrm{G}$ and also that $E_{\mathrm{e}, \max } \sim 45 \mathrm{TeV}$. For these values, electron cooling is important and the escaping particles have a broken power-law distribution which gives the correct shape for the extended $\gamma$-ray spectrum. However, the Xray flux level is then overestimated by a factor of 15 and one should use the so-called magnetic filling factor to obtain the correct flux level. This however cannot be done in our model since the emitted radiation is calculated self-consistently from the particle losses. We thus see that a synchrotron-IC model cannot explain at the same time both the relative flux levels and the relative position of the peak frequencies. The possibility that the $\gamma$-ray emission is due to bremsstrahlung radiation can most probably be ruled out, since the ambient hydrogen density required to fit the spectrum is $n \sim 300 \mathrm{~cm}^{-3}$, a value that is too high. We conclude that the observed radiation from this remnant cannot be of purely leptonic origin.

We thus consider that protons are also accelerated in the remnant. As a secondary electron population produced from charged pion decay cannot explain the X-ray emission (Mastichiadis 1996), primary electrons are again required. However, in this case both electrons and protons contribute to the $\gamma$-ray regime. The inclusion of a relativistic proton population introduces one more free parameter, the energy content in protons $\mathcal{E}_{\mathrm{p}}$ (related to $\bar{Q}_{\mathrm{o}}$ ). For the minimum proton energy we adopt the value $E_{\mathrm{p}, \min }=2 \mathrm{GeV}$ while the maximum proton energy is determined from Eq. (14). For the emission from neutral pions we use a $\delta$-function approximation.

We fit our model to the multi-wavelength spectrum of RX J1713.7-3946 for the parameters $r, \delta, B, E_{\mathrm{e}, \max }, Q_{\mathrm{o}}$ and $\bar{Q}_{\mathrm{o}}$. The resulting theoretical spectrum is shown in Fig. 2 together with the observational data. Although there is a small disagreement with the first two HESS points (at $215 \mathrm{GeV}$ and $255 \mathrm{GeV}$ ), the fit is quite satisfactory and has a chi square $\chi^{2} \simeq 23$ with 22 degrees of freedom (when the last HESS point at $35 \mathrm{TeV}$ is excluded from the fit). From the derived values of $r=3.8$ and $\delta=0.3$ we can calculate the two particle power-law indices $s_{1}=1.77$ and $s_{2}=2.07$. The value of $r$ implies a strong shock and the value of $\delta$ a nearly Kolmogorov-type diffusion $K(p) \propto p^{0.3}$. The magnetic field has the value $B=15 \mu \mathrm{G}$. From this value and the maximum electron energy $E_{\mathrm{e}, \max }=100 \mathrm{TeV}$ we find that the cooling timescale is $t_{\text {cool }}=500 \mathrm{yr}$ and hence the energy losses in the escape region are marginally important. We also deduce that electrons in the acceleration zone are close to steady-state, $p_{1}\left(t_{\mathrm{SN}}\right)=0.7 p_{\max }$, while those in the escape zone have the broken power-law distribution with the break at $p_{\text {cool }}\left(t_{\mathrm{SN}}\right)=20 \mathrm{TeV} / \mathrm{c}=0.2 p_{\max }$. For the protons the maximum energy at the present day is $160 \mathrm{TeV}$. The normalization constant $Q_{\mathrm{o}}=6 \times 10^{40} \mathrm{~s}^{-1}$ determines the total energy content in electrons from both zones $\mathcal{E}_{\mathrm{e}}=3 \times 10^{47} \mathrm{erg}$. The proton normalization constant is $\bar{Q}_{\mathrm{o}}=2 \times 10^{41} \mathrm{~s}^{-1}$ and the corresponding energy content in protons is $\mathcal{E}_{\mathrm{p}}=2 \times 10^{50} \mathrm{erg}$, dominating the total energy content in relativistic particles, i.e. $\mathcal{E}_{\text {tot }} \simeq \mathcal{E}_{\mathrm{p}}$. The majority of this energy is carried by particles in the escape region. For the typical value of supernova explosion energy of a few times $10^{51}$ erg we find that a fraction $\sim 10 \%$ of it is needed in order to explain the non-thermal emission of RX J1713.7-3946.

From these parameters we find that the diffusion coefficient has the form

$K(p)=3 \times 10^{26}\left(\frac{U_{\mathrm{sh}}}{6 \times 10^{3} \mathrm{~km} \mathrm{~s}^{-1}}\right)^{2}\left(\frac{p}{100 \mathrm{TeV} \mathrm{c}^{-1}}\right)^{0.3} \mathrm{~cm}^{2} \mathrm{~s}^{-1}$

and is greater than the lower limit of Bohm diffusion for all particle momenta less than

$p<170\left(\frac{U_{\mathrm{sh}}}{6 \times 10^{3} \mathrm{~km} \mathrm{~s}^{-1}}\right)^{20 / 7} \mathrm{TeV} \mathrm{c}^{-1}$ 
If we thus adopt for the shock velocity the value $U_{\mathrm{sh}}=6 \times$ $10^{3} \mathrm{~km} \mathrm{~s}^{-1}$ then the diffusion coefficient we derive does not violate the lower limit of Bomh diffusion for any value of particle momentum in our model.

\section{Summary and discussion}

We have attempted a fit to the multi-wavelength spectrum of the supernova remnant RX J1713.7-3946 based on the box model of Drury et al. (1999). According to this, particles diffuse in an energy-dependent region around the shock front of the remnant and are accelerated by the first order Fermi mechanism. The particle distribution functions (both for electrons and protons) come as solutions to two coupled partial differential equations, one characterizing the particles inside the acceleration region and the other the ones that have escaped from it. Consequently, one can calculate the radiated photon spectra by folding the derived particle distribution functions with the single particle emissivity for the radiative processes assumed. For the case of electrons the corresponding radiative processes are synchrotron, IC scattering and bremsstrahlung emission, while protons produce radiation only through the decay of pions.

We found that, within the strict assumptions of the box model, a purely leptonic origin of the radiation cannot explain the observations. The leptonic model is viable only in the case where the magnetic field in the escape region has a value large enough to allow for substantial particle cooling during the lifetime of the remnant. The escaping particles then have a broken power-law distribution that can explain the extended $\gamma$-ray spectrum of RX J1713.7-3946. For such a high value of the magnetic field, the level of X-ray emission significantly exceeds the observed one. We thus reach the same conclusion as all the other leptonic models used for this source, i.e. that we cannot explain at the same time the relative position of the synchrotron and IC peaks and their relative fluxes, unless we use the filling factor. However the above conclusions are valid under the assumptions of a spatially averaged model and a plane-parallel shock geometry. In a model where the spatial dependence is treated in detail one could include other effects like variable injection rate at different supernova radii and/or expansion losses, although the error from the latter should not be important. Whether a purely leptonic model would be more successful in such a case remains to be investigated.

The inclusion of the relativistic hadronic population provides an additional component in $\gamma$-rays and a satisfactory fit can then be obtained. However, the hadronic component can be treated only approximately in our model since the test particle approximation that we used is not strictly valid and the shock becomes modified by the hadrons. To take this into account one should use calculations based on numerical models.

From the fit to the spectrum of RX J1713.7-3946 we also deduced the dependence of the diffusion coefficient on energy. We found that a nearly Kolmogorov-type diffusion coefficient $(\delta=0.3)$ can reproduce the observed spectrum quite well. The $1 \sigma$ estimate for the parameter $\delta$ is well concentrated around this value, $0.1 \lesssim \delta \lesssim 0.5$. This is because a change in $\delta$ mostly influences the maximum electron and proton energies and these are well defined from the observed spectrum. This range of $\delta$ marginally allows for a Kraichnan-type turbulence spectrum, while the case of Bohm diffusion is not favored by our results.

We conclude that the model we used, despite certain assumptions, has two main advantages over other models, in that a) the particle distribution functions are calculated from a theoretical model and are not assumed a priori and b) it can give a method to estimate the energy dependence of the diffusion coefficient in supernova remnants.

Acknowledgements. The authors would like to thank the anonymous referee for valuable comments as well as F. Aharonian for useful discussions and N. Vlahakis for making comments that helped improve the manuscript. The project is co-funded by the European Social Fund and National Resources (EPEAEK II) PYTHAGORAS.

\section{References}

Aharonian, F. A., Akhperjanian, A. G., Aye, K.-M., et al. 2004, Nature, 432, 75 Aharonian, F. A., Akhperjanian, A. G., Bazer-Bachi, A. R., et al. 2006, A\&A, 449,223

Aharonian, F. A., \& Atoyan, A. M. 1999, A\&A, 351, 330

Ball, L., \& Kirk, J. G. 1992, ApJ, 396, L39

Bell, A. R. 1978, MNRAS, 182, 147

Berezhko, E. G., Ksenofontov, L. T., \& Völk, H. J. 2002, A\&A, 395, 943

Berezhko, E. G., \& Völk, H. J. 2006, A\&A, 451, 981

Blandford, R. D., \& Ostriker, J. P. 1978, ApJ, 221, L29

Dorfi, E. A. 1991, A\&A, 251, 597

Drury, L. O., Aharonian, F. A., \& Völk, H. J. 1994, A\&A, 287, 959

Drury, L. O., Duffy, P., Eichler, D., \& Mastichiadis, A. 1999, A\&A, 347, 370

Ellison, D. C., Slane, P., \& Gaensler, B. M. 2001, ApJ, 563, 191

Enomoto, R., Tanimori, T., Naito, T., et al. 2002, Nature, 416, 823

Kirk, J. G., Melrose, D. B., \& Priest, E. R. 1994, Plasma Astrophysics, Saas Fee Advanved Course 24, ed. A. O. Benz, \& T. J.-L. Courvoisier (Berlin: Springer-Verlag)

Koyama, K., Kinugasa, K., Matsuzaki, K., et al. 1997, PASJ, 49, L7

Krymskii, G. F. 1977, Akademiia Nauk SSSR Doklady, 234, 1306

Lazendic, J. S., Slane, P. O., Gaensler, B. M., et al. 2004, ApJ, 602, 271

Mastichiadis, A. 1996, A\&A, 305, L53

Muraishi, H., Tanimori, T., Yanagita, S., et al. 2000, A\&A, 354, L57

Pannuti, T. G., Allen, G. E., Houck, J. C., \& Sturner, S. J. 2003, ApJ, 593, 377

Pfeffermann, E., \& Aschenbach, B. 1996, in Roentgenstrahlung from the Universe, 267

Pohl, M. 1996, A\&A, 307, L57

Reimer, O., \& Pohl, M. 2002, A\&A, 390, L43

Reynolds, S. P., \& Keohane, J. W. 1999, ApJ, 525, 368

Slane, P., Gaensler, B. M., Dame, T. M., et al. 1999, ApJ, 525, 357

Tomida, H. 1999, Ph.D. Thesis, Kyoto University

Uchiyama, Y., Aharonian, F. A., \& Takahashi, T. 2003, A\&A, 400, 567

Wang, Z. R., Qu, Q.-Y., \& Chen, Y. 1997, A\&A, 318, L59

Weekes, T. C., Badran, H., Biller, S. D., et al. 2002, APh, 17, 221 